\title{
Assessment of Ki-67 for Predicting Effective Prognosis in Breast Cancer Subtypes
}

\author{
Sangjung Park ${ }^{1, \S}$, Sunyoung Park ${ }^{2, \S}$, Jungho Kim², Sungwoo Ahn², \\ Kwang Hwa Park ${ }^{3, \dagger}$ and Hyeyoung Lee ${ }^{2, \dagger}$ \\ ${ }^{1}$ Department of Biomedical Laboratory Science, College of Life and Health Sciences, \\ Hoseo University, Asan 31499, Korea \\ ${ }^{2}$ Department of Biomedical Laboratory Science, College of Health Sciences, \\ Yonsei University, Wonju 26493, Korea \\ ${ }^{3}$ Department of Pathology, College of Medicine, Yonsei University Wonju, Wonju 26426, Korea
}

Ki-67 has been widely performed and become an important biomarker in worldwide clinics, but the standard cut off value of Ki-67 index in breast cancer is still controversy. The objective study was to understand the Ki-67 in breast cancer subtypes and to investigate relative risk of breast cancer subtypes according to Ki-67 cut off value in Korean breast cancer. Immunohistochemical staining (IHC) for estrogen receptor (ER), progesterone receptor (PR), human epidermal growth factor receptor 2 (HER2), and Ki-67 index was examined from 123 breast cancer patients. Ki-67 index was significantly overexpressed in PR, ER, and HER2 hormone negative groups. Ki-67 index in Triple negative and HER2 subtypes was shown significantly higher than that in Luminal A and Luminal B subtype. Then, we compared the relative risk of each subtype according to $14 \%$ and $20 \% \mathrm{Ki}-67$ cut off value, which were applied in most clinics. Especially, 20\% Ki-67 cut off value in HER2 and Triple negative subtypes was shown 8.41 fold and 2.83 fold higher relative risk than this in Luminal A subtype. Moreover, Ki-67 index in HER2 2+ or 3+ status showed significantly overexpressed than this in HER2 $1+$ status. At the $20 \%$ Ki-67 cut off value, HER2 $1+$ or 2+ status and 3+ status showed significant difference. Therefore, the $20 \% \mathrm{Ki}-67$ cut off value will be useful as a precise prognostic management and helpful for interpreting diverse outcomes of other subtypes in breast cancer patients.

Key Words: Ki-67, Breast cancer, Triple negative, HER2, Immunohistochemistry

\section{INTRODUCTION}

Breast cancer has the second most incidence rates in females (Siegel et al., 2016). Treatment of breast cancer is determined by breast cancer subtypes according to main distinct hormone (estrogen and progesterone) receptors and human epidermal growth factor receptor 2 receptor (HER2) (O'Brien et al., 2010; Luporsi et al., 2012). It's classified as four subtypes; Luminal A subtype, which shows Estrogen

* Received: November 29, 2017 / Revised: December 22, 2017 / Accepted: January 24, 2018

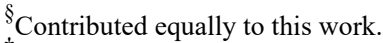

Corresponding author: Kwang Hwa Park. Department of Pathology, College of Medicine, Yonsei University Wonju, 20 Ilsan-ro, Wonju-si, Gangwon-do 26426, Korea.

Tel: +82-33-741-1556, Fax: +82-33-731-6590, e-mail: abba@yonsei.ac.kr

${ }^{\dagger}$ Corresponding author: Hyeyoung Lee. Department of Biomedical Laboratory Science, College of Health Sciences, Yonsei University, 1 Yonseidae-gil, Wonju, Gangwon 26426, Korea.

Tel: +82-33-760-2740, Fax: +82-33-760-2561, e-mail: hyelee@yonsei.ac.kr

(C) The Korean Society for Biomedical Laboratory Sciences. All rights reserved.

(c) This is an Open Access article distributed under the terms of the Creative Commons Attribution Non-Commercial License (http://creativecommons.org/licenses/by-nc/3.0/) which permits unrestricted non-commercial use, distribution, and reproduction in any medium, provided the original work is properly cited. 
receptor (ER) or progesterone receptor (PR)-positive and HER2-negative breast cancer; Luminal B subtype, which shows ER or PR-positive and HER2-positive breast cancer; HER2 subtype, which shows ER or PR-negative and HER2positive breast cancer; Triple negative, which shows ER or PR-negative and HER2 negative subtype.

As a requirement for the effectiveness and improvement of breast cancer treatment, nowadays, St. Gallen Consensus suggested the revised classification of breast cancer subtypes adding Ki-67 marker proposed by Cheang et al. (Cheang et al., 2009; Goldhirsch et al., 2011). Among the four subtypes, Luminal B, HER2, and Triple negative subtype patients have shown poorer outcomes than Luminal A subtype patients. In the revised classification, $\mathrm{Ki}-67$ index is more surrogate marker to differentiate Luminal A and Luminal B subtypes in the perspective of prediction to poor prognosis (O'Brien et al., 2010; Leong and Zhuang, 2011).

$\mathrm{Ki}-67$ is a nuclear protein that is associated with cell proliferation and considered as prognostic markers in breast cancer (Trihia et al., 2003). Ki-67 index is ranged from 0\% to $100 \%$ and measured by the percentage of the positive stained tumor nuclear cells out of all tumor cells (Trihia et al., 2003). However, the Ki-67 index for differentiating high and low Ki-67 is controversial (Cheang et al., 2009; Bustreo et al., 2016; Chung et al., 2016). As a cut-off value for differentiating Ki-67 high and Ki-67 low, 14\% and 20\% were both used in pathologic fields. Even though Ki-67 is suggested as an important marker in breast cancer, the implication of clinical decision and prediction is needed to be accurate.

The purpose of this study is to find out Ki-67 characteristics of four subtypes in 123 Korean breast cancer patients. We focused on the characteristics of Ki-67 index in ER, PR and HER2 IHC assessment. We also analyzed relative risk of each breast cancer subtypes according to $\mathrm{Ki}-67$ cut-off value (14\% and 20\%) for effective decision-making.

\section{MATERIALS AND METHODS}

\section{Study population}

A total of 123 formalin-fixed, paraffin-embedded (FFPE) tissues from invasive ductal carcinoma were collected from the department of pathology, Yonsei University Wonju
Severance Christian Hospital in Republic of Korea between 2011 and 2013. Ethical approval for this study was obtained from the Ethical Committee of our Institution (approval no. 1-2010-0018).

\section{Immunohistochemistry (IHC) staining}

The 123 FFPE tissues were examined with immunohistochemistry for ER, PR, and HER2 and were performed with Ki-67 according to the manufacturer's protocol (Dako Cytomation, Glostrup, Denmark). The slides of 123 FFPE tissues were reviewed separately by 2 qualified pathologists. To estimate the percentage of cells that stained positive for ER and PR, 100 tumor cells were counted, and the ratio of the number of stained cells to the total number of cells was calculated and reported as a percentage. For ER and PR, above $10 \%$ of the proportion and intensity scores was considered as ER and PR positive. For HER2, HER2 0/1+ indicate HER2-negative status and HER2 3+ indicate HER2-positive status. Tumors showing a $2+$ score by IHC are considered undetermined and tested by FISH to confirm their HER2 status (Moerland et al., 2006). Ki-67 index is defined as positivity rates of stained malignant cells and is ranged from $0 \%$ to $100 \%$.

Overall, breast carcinomas were divided into 4 major molecular subtypes: Luminal A, Luminal B, Triple negative, and HER2.

\section{Statistical analysis}

Descriptive statistics and $t$-test of Ki-67 index in ER, PR and HER2 positive and negative patients were analyzed using GraphPad Prism v5.02 (GraphPad, La Jolla, CA, USA). For Ki-67 index in Luminal B, HER2, and Triple negative expression compared to Luminal A, Student's $t$-test was used to determine statistical significance. The relative risk for four subtypes and HER2 grade according to the Ki-67 index of $14 \%$ and $20 \%$ was calculated by MedCalc software (MedCalc Software, Mariakerke, Belgium).

\section{RESULTS}

\section{Clinical characteristics in breast cancer}

Patient characteristics are summarized in Table 1 . One 
Table 1. Clinical characteristics in 123 breast cancer women

\begin{tabular}{lcc}
\hline \hline Variables & Cases & $\%$ \\
\hline Age & 12 & \\
$30 \mathrm{~s}$ & 38 & 30.8 \\
$40 \mathrm{~s}$ & 42 & 34.1 \\
$50 \mathrm{~s}$ & 19 & 15.4 \\
$60 \mathrm{~s}$ & 12 & 9.8 \\
$>70 \mathrm{~s}$ & & \\
ER IHC & 41 & 33.3 \\
Negative & 82 & 66.7 \\
Positive & & \\
PR IHC & 53 & 43.1 \\
Negative & 70 & 56.9 \\
Positive & & \\
HER2 IHC & & 35.8 \\
Negative & 44 & 64.2 \\
Positive & 79 & \\
\hline
\end{tabular}

ER, Estrogen receptor; PR, Progesterone receptor; HER2, Human epidermal growth factor receptor 2; IHC, Immunohistochemistry

hundred and twenty-three FFPE tissue samples were used in this study. Cases were randomly collected according to Korean breast cancer prevalence according to age distribution, followed as 12 cases (9.8\%) in $30 \mathrm{~s}, 38$ cases (30.9\%) in $40 \mathrm{~s}$, 42 cases (34.1\%) in $50 \mathrm{~s}, 19$ cases (15.4\%) in $60 \mathrm{~s}$, and 12 cases $(9.8 \%)$ over $70 \mathrm{~s}$. ER IHC positive cases were 82 (66.7\%). PR IHC positive cases were 70 (56.9\%). HER2 IHC positive cases were $79(64.2 \%)$ (Table 1).

\section{Ki-67 index on HER2, estrogen, and progesterone status}

Ki-67 index in ER, PR, and HER2 negative cancer was significantly higher than in those in ER, PR, and HER2 positive cancer, respectively $(P<0.0001, P<0.0001$, and $P=$ 0.0006). The mean of Ki-67 index was 53.05 (95\% Confidence Interval (CI) 44.30 61.80) in ER negative and 21.33 (95\% CI 17.93 24.73) in ER positive. The mean of Ki-67 index was 45.94 (95\% CI 38.17 53.72) in PR negative and 21.27 (95\% CI 17.43 25.11) in PR positive. The mean of Ki-67 index was 42.23 (95\% CI 33.58 50.87) in HER2 negative and 26.15 (95\% CI 21.37 30.93) in HER2 positive (Table 2).
Table 2. Ki-67 index on HER2, estrogen, and progesterone IHC

\begin{tabular}{lcccc}
\hline \hline & Cases & \multicolumn{3}{c}{ Ki-67 index } \\
\cline { 3 - 5 } & & Mean & $(95 \% \mathrm{CI})$ & $P$-value \\
\hline ER IHC & & & & \\
Negative & 41 & 53.05 & $(44.30 \sim 61.80)$ & $<0.0001$ \\
Positive & 82 & 21.33 & $(17.93 \sim 24.73)$ & \\
PR IHC & & & & \\
Negative & 53 & 45.94 & $(38.17 \sim 53.72)$ & $<0.0001$ \\
Positive & 70 & 21.27 & $(17.43 \sim 25.11)$ & \\
HER2 IHC & & & & \\
Negative & 44 & 42.23 & $(33.58 \sim 50.87)$ & 0.0006 \\
Positive & 79 & 26.15 & $(21.37 \sim 30.93)$ & \\
\hline
\end{tabular}

ER, Estrogen receptor; PR, Progesterone receptor; HER2, Human epidermal growth factor receptor 2

\section{Comparison between the Ki-67 index and four subtypes of breast cancer}

In order to investigate $\mathrm{Ki}-67$ index in each breast cancer subtype, 123 breast cancer tissues were divided into four groups; Luminal A, Luminal B, HER2, and Triple negative by using ER, PR, and HER2 status. The mean of Ki-67 index in Triple negative breast cancer subtype was 59.38 (95\% CI 30.00 83.75) and followed by $42.13(95 \% \mathrm{CI}$ 26.25 63.75) in HER2, 24.06 (95\% CI 10.00 30.00) in Luminal B, and 20.15 (95\% CI 8.50 30.00) in Luminal A. Compared to Ki-67 in Luminal A which expects better prognosis, Ki-67 in HER2 and Triple negative breast cancer subtype was shown significantly higher expression, respectively $(P<0.0001$ and $P<0.0001)$ (Fig. 1).

In order to investigate whether cut-off of Ki-67 in breast cancer subtypes is affected, $14 \%$ cut off and $20 \%$ cut off of $\mathrm{Ki}-67$ in each breast cancer subtype was analyzed by relative risk. Relative risks of $14 \%$ and $20 \%$ cut off Ki-67 in Luminal $B$ were not shown significant difference compared to those in Luminal A. A relative risk of $14 \%$ cut off Ki-67 in HER2 and Triple negative were 5.43 (95\% CI 0.77 38.01) and 2.51 (95\% CI 0.84 7.47), but no significant difference was shown ( $P=0.09$ and $P=0.09$ ). Notably, Relative risk of $20 \%$ cut off Ki-67 in HER2 and Triple negative subtypes were 8.41 (95\% CI 1.19 59.67) and 2.83 (95\% CI 1.08 7.38) ( $P=0.03$ and $P=0.03$, respectively) (Table 3 ). 


\section{Comparison of the Ki-67 index in HER2 positive status}

Since Ki-67 in HER2 subtype was shown high expression, we found out HER2 is related to Ki-67. Ki-67 in HER2 1+, HER2 2+ and HER2 3+ were compared. Ki-67 in HER2 $2+$ and HER2 $3+$ patients showed significantly higher expression, compared to that in HER2 $1+(P=0.0485$ and $P=$ 0.004). The mean of Ki-67 was a 19.07 (95\% CI 11.42 26.71) in HER2 1+, 27.58 (95\% CI 18.41 36.76) in HER2 2+, and 32.22 (95\% CI 23.82 40.62) in HER 3+ (Fig. 2).

In order to analyze clinical implication of a $14 \%$ cut off $\mathrm{Ki}-67$ and 20\% cut-off Ki-67 in HER2 status, the relative risk

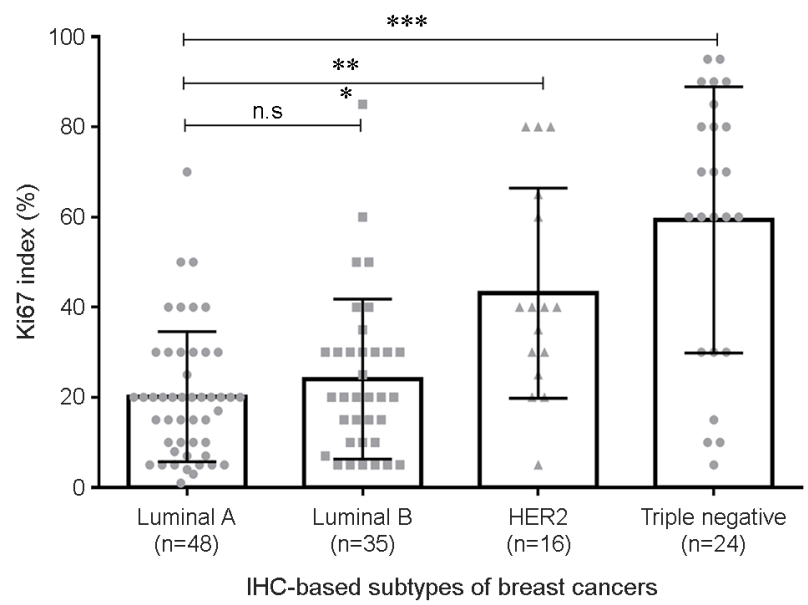

Fig. 1. Comparison between the Ki-67 expression levels and four subtypes of breast cancer. The high expression of the Ki-67 in basal-like breast cancer subtype was the highest expressed and followed by HER2 and luminal B. Compared to Ki-67 in luminal A, Ki-67 in HER2 and basal-like breast cancer subtype was shown significantly high expression, respectively $(P<0.0001$ and $P<$ $0.0001)$. *, $P<0.05$; **, $P<0.01 ; * * *, P<0.001$. was analyzed. 20\% cut-off Ki-67 in HER2 3+ is 2.23 (95\% CI 1.13 4.84) higher than HER2 $1+(P=0.02)$ (Table 4).

\section{DISCUSSION}

Prognostic markers are important for treatment in various cancers and $\mathrm{Ki}-67$ index in breast cancer has been widely used as a perspective of predictive and prognostic values (Kim et al., 2008; Kim et al., 2016). However, the cut-off value of $\mathrm{Ki}-67$ is varied and need standard predictive or prognostic value. Especially, several studies focused on distinction between Luminal A and B breast cancer subtypes

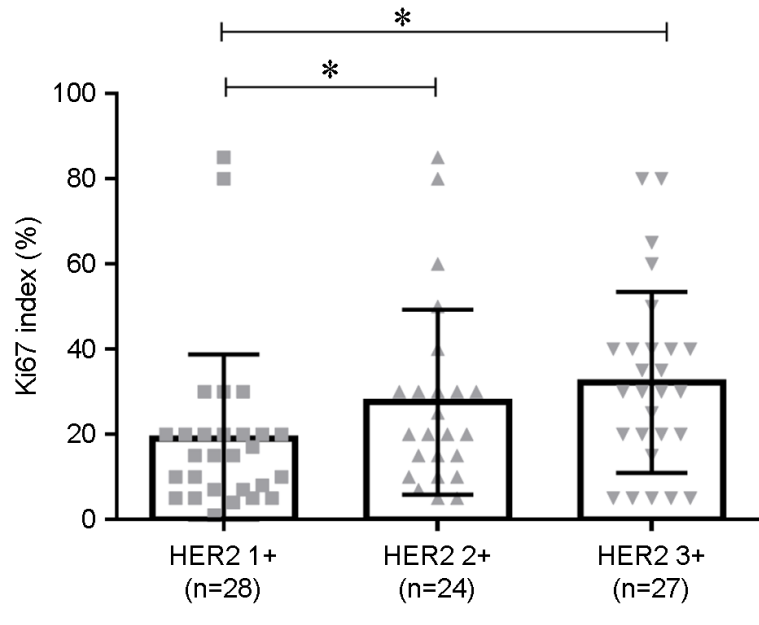

HER2 IHC

Fig. 2. Comparison of the Ki-67 expression in HER2 positive status. Ki-67 in HER2 2+ and HER2 3+ was significantly shown higher expression compared to Ki-67 in HER2 $1+(P=0.0485$ and $P=0.004)$. The mean of Ki-67 was $19.07(95 \%$ CI $11.42 \sim 26.71)$ in HER2 1+, 27.58 (95\% CI 18.41 36.76) in HER2 2+, and 32.22 (95\% CI 23.82 40.62).

Table 3. Relative risk between Ki-67 index and breast cancer subtypes

\begin{tabular}{|c|c|c|c|c|}
\hline & \multicolumn{2}{|c|}{$\mathrm{Ki}-67$ index $\geq 14 \%$} & \multicolumn{2}{|c|}{$\mathrm{Ki}-67$ index $\geq 20 \%$} \\
\hline & Relative risk $(95 \% \mathrm{CI})$ & $P$-value & Relative risk $(95 \% \mathrm{CI})$ & $P$-value \\
\hline Luminal A $(n=48)$ & ref & & ref & \\
\hline Luminal B $(\mathrm{n}=35)$ & $1.14(0.65 \sim 2.01)$ & $P=0.65$ & $1.15(0.68 \sim 1.93)$ & $P=0.60$ \\
\hline $\operatorname{HER} 2(\mathrm{n}=16)$ & $5.43(0.77 \sim 38.01)$ & $P=0.09$ & $8.41(1.19 \sim 59.67)$ & $P=0.03$ \\
\hline Triple negative $(\mathrm{n}=24)$ & $2.51(0.84 \sim 7.47)$ & $P=0.09$ & $2.83(1.08 \sim 7.38)$ & $P=0.03$ \\
\hline
\end{tabular}

Luminal A, ER positive or PR positive, and HER2 negative; Luminal B, ER positive or PR positive, and HER2 positive; HER2, ER negative, PR negative, and HER2 positive; Triple negative, ER negative, PR negative, and HER2 negative 
Table 4. Relative risk between Ki-67 index and HER 2 positive breast cancer patients

\begin{tabular}{lccccc}
\hline \hline & \multicolumn{2}{c}{ Ki-67 index $\geq 14 \%$} & & \multicolumn{2}{c}{ Ki-67 index $\geq 20 \%$} \\
\cline { 2 - 2 } & Relative risk (95\% CI) & $P$-value & & Relative risk (95\% CI) & $P$-value \\
\hline HER2 1+ $(\mathrm{n}=28)$ & ref & & ref & \\
HER2 2+ $(\mathrm{n}=24)$ & $1.59(0.77 \sim 3.3)$ & $P=0.21$ & & $1.46(0.74 \sim 2.87)$ & $P=0.27$ \\
HER2 3+ $(\mathrm{n}=27)$ & $1.96(0.90 \sim 4.31)$ & $P=0.09$ & & $2.23(1.13 \sim 4.84)$ & $P=0.02$ \\
\hline
\end{tabular}

(Cheang et al., 2009). The re-classification by Ki-67 as the prognostic point of view became important because a Luminal B subtype has shown poorer outcomes than Luminal A subtype (Cheang et al., 2009; Goldhirsch et al., 2011).

Ki-67 index is highly expressed in all three ER, PR and HER2 negative status. Ki-67 index in ER, PR, and HER2 negative breast cancer was 2.5, 2.1, and 1.6 fold higher than those in ER, PR, and HER2 positive breast cancer $(P<$ 0.0001, $P<0.0001$, and $P=0.0006$ ) (Table 2). Moreover, the expression of the Ki-67 in Triple negative breast cancer subtype was the highest expressed and followed by HER2 and Luminal B (Fig. 1).

Jian Yan et al. indicated prognostic values of two groups with each subtype using ER, PR, and HER2 status, and Ki-67 positive and negative. Compared to Luminal A, Luminal B, HER2, and Triple negative breast cancer subtype showed significant poor prognosis. Moreover, the Ki-67 positive breast cancer was shown poorer prognosis than $\mathrm{Ki}-67$ negative breast cancer (Yan et al., 2015). This study supports our data and Ki-67 index is routinely needed with Triple negative and HER2 subtype to differentiate poor prognosis.

Even though Ki-67 index is commonly used worldwide, standard cut-off value for Ki-67 index was not determined. For effective treatment of breast cancer, controversially used cut off Ki-67, which is $14 \%$ and $20 \%$, were analyzed. A relative risk of $20 \%$ cut off $\mathrm{Ki}-67$ in HER2 and basal like is 8.41 and 2.83 and showed better relative risk compared to $14 \%$ cut off Ki-67 (Table 3). Tashima et al. showed that optimal cut off $20 \% \mathrm{Ki}-67$ in basal like cancer indicated poor prognosis through retrospective study of 4,000 breast cancer patients (Tashima et al., 2015). Bustreo et al. suggested over $20 \% \mathrm{Ki}-67$ in luminal subtypes also showed poor prognosis (Bustreo et al., 2016). Denkert et al. and Tan et al. suggested
$20 \%$ cut off value of $\mathrm{Ki}-67$ impact the poor prognosis and chemotherapy treatment in breast cancer (Denkert and von Minckwitz, 2014; Tan et al., 2014). In this study, we also support that $20 \% \mathrm{Ki}-67$ cut off value is reliable to predict prognosis of breast cancer in Korea.

Another new finding was that $\mathrm{Ki}-67$ index according to HER2 status was significantly increased (HER2 1+ vs HER2 $2+$ and HER2 $1+$ vs HER2 $3+, P=0.0485$ and $P=0.004)$ (Fig. 2). Shokouh et al. also found that Ki-67 is related to not only tumor grade but also HER2 status (Shokouh et al., 2015). Our data also showed similar result and co-monitoring of Ki-67 and HER2 as well as Luminal A and B will play a clinically crucial role in patients with ambiguous HER2 status as additional prognostic marker.

In summary, we compared clinical risk of $\mathrm{Ki}-67$ using $14 \%$ and $20 \%$ cut off value in four breast cancer subtypes and found that $20 \%$ cut off value is more practical to apply clinical making decision in Korea. In addition, we individually confirmed relation between Ki-67 index and other conventional markers; ER, PR, and HER2 receptors to find out clinical management of Ki-67 to provide complementary guideline for chemotherapy and hormonal therapy.

\section{ACKNOWLEDGEMENTS}

None.

\section{CONFLICT OF INTEREST}

None.

\section{REFERENCES}

Bustreo S, Osella-Abate S, Cassoni P, Donadio M, Airoldi M, Pedani F, Papotti M, Sapino A, Castellano I. Optimal Ki-67 
cut-off for luminal breast cancer prognostic evaluation: A large case series study with a long-term follow-up. Breast Cancer Research Treatment. 2016. 157: 363-371.

Cheang MC, Chia SK, Voduc D, Gao D, Leung S, Snider J, Watson M, Davies S, Bernard PS, Parker JS, Perou CM, Ellis MJ, Nielsen TO. Ki-67 index, her2 status, and prognosis of patients with luminal B breast cancer. Journal of National Cancer Institute. 2009. 101: 736-750.

Chung YR, Jang MH, Park SY, Gong G, Jung WH, Korean Breast Pathology Ki-67 Study G. Interobserver variability of Ki-67 measurement in breast cancer. Journal of Pathology and Translational Medicine. 2016. 50: 129-137.

Denkert C, von Minckwitz G. Reply to Ki-67 in breast cancer: A useful prognostic marker! Annals of Oncology. 2014. 25: 542 $-543$.

Goldhirsch A, Wood WC, Coates AS, Gelber RD, Thurlimann B, Senn HJ, Panel M. Strategies for subtypes--dealing with the diversity of breast cancer: Highlights of the St. Gallen international expert consensus on the primary therapy of early breast cancer 2011. Annals of Oncology. 2011. 22: 1736-1747.

Kim TJ, Kim SC. Analysis of the bcl-2, Ki-67 and p53 expression level based on the Gleason score group of prostate adenocarcinoma. Biomedical Science Letter. 2008. 14: 156-165.

Kim Y, Seok JY, Hyun KY, Lee GH, Choi SC. Correlation of Glasgow pronostic score or procalcitonin to clinical variables in patients with pretreatment lung cancer. Biomedical Science Letter. 2016. 22: 9-17.

Leong AS, Zhuang $\mathrm{Z}$. The changing role of pathology in breast cancer diagnosis and treatment. Pathobiology. 2011. 78: 99 -114 .

Luporsi E, Andre F, Spyratos F, Martin PM, Jacquemier J, PenaultLlorca F, Tubiana-Mathieu N, Sigal-Zafrani B, Arnould L, Gompel A, Egele C, Poulet B, Clough KB, Crouet H, Fourquet A, Lefranc JP, Mathelin C, Rouyer N, Serin D, Spielmann M, Haugh M, Chenard MP, Brain E, Cremoux P, Bellocq JP. Ki-67: Level of evidence and methodological considerations for its role in the clinical management of breast cancer: Analytical and critical review. Breast Cancer Research Treatment. 2012. 132: 895-915.

Moerland E, Van Hezik RL, Van Der Aa TC, Van Beek MW, Van Den Brule AJ. Detection of her2 amplification in breast carcinomas: Comparison of multiplex ligation-dependent probe amplification (MLPA) and fluorescence in situ hybridization (FISH) combined with automated spot counting. Cellular Oncology. 2006. 28: 151-159.

O'Brien KM, Cole SR, Tse CK, Perou CM, Carey LA, Foulkes WD, Dressler LG, Geradts J, Millikan RC. Intrinsic breast tumor subtypes, race, and long-term survival in the carolina breast cancer study. Clinical Cancer Research. 2010. 16: 6100-6110.

Shokouh TZ, Ezatollah A, Barand P. Interrelationships between $\mathrm{Ki}-67$, her2/neu, p53, er, and pr status and their associations with tumor grade and lymph node involvement in breast carcinoma subtypes retrospective-observational analytical study. Medicine. 2015. 94: e1359.

Siegel RL, Miller KD, Jemal A. Cancer statistics, 2016. CA: A Cancer Journal for Clinicians. 2016. 66: 7-30.

Tan QX, Qin QH, Yang WP, Mo QG, Wei CY. Prognostic value of $\mathrm{Ki}-67$ expression in HR-negative breast cancer before and after neoadjuvant chemotherapy. International Journal of Clinical and Experimental Pathology. 2014. 7: 6862-6870.

Tashima R, Nishimura R, Osako T, Nishiyama Y, Okumura Y, Nakano M, Fujisue M, Toyozumi Y, Arima N. Evaluation of an optimal cut-off point for the $\mathrm{Ki}-67$ index as a prognostic factor in primary breast cancer: A retrospective study. PLOS One. 2015. 10: e0119565.

Trihia H, Murray S, Price K, Gelber RD, Golouh R, Goldhirsch A, Coates AS, Collins J, Castiglione-Gertsch M, Gusterson BA, International Breast Cancer Study G. Ki-67 expression in breast carcinoma: Its association with grading systems, clinical parameters, and other prognostic factors-a surrogate marker? Cancer. 2003. 97: 1321-1331.

Yan J, Liu XL, Han LZ, Xiao G, Li NL, Deng YN, Yin LC, Ling LJ, Yu XY, Tan CL, Huang XP, Liu LX. Relation between Ki-67, ER, PR, HER2/Neu, p21, EGFR, and Top II-alpha expression in invasive ductal breast cancer patients and correlations with prognosis. Asian Pacific Journal of Cancer Prevention. 2015. 16: 823-829.

https://doi.org/10.15616/BSL.2018.24.1.9

Cite this article as: Park S, Park S, Kim J, Ahn S, Park KH, Lee HY. Assessment of Ki-67 for Predicting Effective Prognosis in Breast Cancer Subtypes. Biomedical Science Letters. 2018. 24: 9-14. 